\title{
Solar Eclipses and Public Education
}

\author{
By J. M. Pasachoff
}

Williams College-Hopkins Observatory, Williamstown, MA 01267, USA

Solar eclipses draw the attention of the general public to celestial events in the countries from which they are visible, and broad public education programs are necessary to promote safe observations. Most recently, a subcommittee of IAU Commission 46 composed of Julieta Fierro (from the National University of Mexico), the Canadian professor of optometry Ralph Chou (from the University of Waterloo) and me provided information about safe observations of the 24 October 1995 eclipse to people in Pakistan, India, Cambodia, Vietnam, and Guam. An important point is that there are advantages to seeing eclipses, including inspiration to students, and that people must always be given correct information. If scare techniques are used to warn people off eclipses, when it is later found out that the eclipse was not dangerous and, indeed, was spectacular, these students and other individuals will not trust warnings for truly hazardous activities like smoking, drugs, and behavior that puts one at risk for AIDS.

A total eclipse of the Sun is the most spectacular sight that can be seen, in my view, both from its physical and from its emotional impact, with the otherwise powerful Sun disappearing in the middle of the day. Though public interest in eclipses may be intense for only the immediate days preceding them, we can nonetheless take advantage of this interest to carry across important scientific ideas. The notion that the Universe is understandable and, in important ways, predictable, is a powerful idea that acts against the ideas of superstition and pseudoscience that are so rampant. Though I certainly think the diamond ring effect and totality are spectacular, I am most moved at first contact, when the moon barely kisses the Sun, because then it becomes clear that the predictions of astronomers were correct, and that I have not been led by a hoax into a wild-goose chase.

It is interesting to note that no fewer than three papers at this IAU Colloquium are devoted to public education through solar eclipses, those of Nirupama Raghavan from India and Rute Helena Trevisan from Brazil, as well as my own. Indeed, Dr. Raghavan reported that over 150 million people had the results of her educational intervention. The 1991 eclipse went over Mexico City; the 1995 eclipse went over Calcutta; and the 1999 eclipse will go over several major cities in Europe, so it is common for large numbers of people to become interested in what is going on in the sky, and how to watch it safely. Television is not an adequate substitute for viewing an eclipse directly, but we must work to make sure that people appreciate that truth.

We astronomers must be out in front in providing useful and correct information. All too often, the impression is spread early on, months before the eclipse, that eclipses are hazardous and that it is easy to go blind from watching it. The kernel of truth in that report is often overwhelmed by overbearing scare techniques. That is one reason that when I formed a subcommittee of Commission 46 on the Teaching of Astronomy of the IAU, I was glad to include Prof. Ralph Chou, professor of optometry at the University of Waterloo in Canada, and an expert on eclipse filters and eye safety. Prof. Julieta Fierro of the National University in Mexico City joined us on this subcommittee, bringing her experience in public education at the favorable total solar eclipse that graced her country in 1991.

In fact, it is not as easy to damage your eyes by looking at the Sun as many people assume. On a normal day, the eye blink reflex keeps you from staring at the Sun, while in 
the minutes before totality the total flux of sunlight may not activate this reflex though the specific intensity of the solar photosphere remains only slightly diminished (by limb darkening) from that at the center of the solar disk. Still, a brief glance at the Sun does not harm, just as people's field of view often crosses the Sun by accident on a normal day at the beach. What I like to emphasize is the following idea: never stare at the Sun, not at an eclipse and not on a normal day. Even when I view the partial phases of an eclipse with a suitable filter, I never look for more than a couple of seconds, and then I look away for a while. And one must always be careful when looking at the Sun with any optical aid, such as a telescope or binoculars, because the focused Sun is much more harmful than the unfocused Sun.

The number of cases of eye damage one hears of is very small, especially given the huge numbers of people who watch eclipses. In fact, if one were to survey a large section of the population, one would find damage in many eyes, and one wonders if most of the reports of eye damage were really caused by the eclipse. After the annular eclipse in 1984 in the United States, after which newspapers reported eye damage to a girl in Racine, Wisconsin, who had stared at the Sun for $\mathbf{4 5}$ minutes-something unusual and difficult to do even during an eclipse-we checked up months later and found that she had almost entirely recovered. So I ask readers, when they hear of eclipse eye damage in their own country, to try to check up on the actual circumstances and on the eventual results.

Public education programs can be valuable, and the opportunity of an eclipse leads local newspapers to run many eclipse-related articles. Since the 1980 eclipse in India, enough representations have been made by Indian scientists and educators that large numbers of Indian citizens were willing to be outdoors, to watch, to eat food, and to carry out other activities during the 1995 eclipse, a change commented on by local newspapers.

Public information programs about eye safety must be carried out for the partial solar eclipse of October 12,1996, and the total solar eclipse of August 11,1999, for both of which most sites are in Europe. Though no totality will occur in Europe in 1996, most people won't travel to the zone of totality in 1999 so would find the $60 \% 1996$ eclipse of equal interest. Thus this year's partial eclipse can act not only as a bonding between European citizens and the sky but also as a trial run for the 1999 eclipse that will receive so much more publicity.

Ignorance of the true nature and value of eclipses has led to unfortunate barring of students from seeing eclipses. I have seen this problem often in the two dozen eclipses of my experience, including such varied sites as Australia in 1974; Manitoba, Canada in 1979; and Virginia, United States, in 1984. What happens when students who were locked in basement classrooms on the opposite side of the building from the Sun get out of school? They might meet a friend who says, "Didn't you see the eclipse, it was beautiful." "No,"they say, "the teacher said we would go blind." When they learn from the friend about the spectacular sight they missed, how will they respond to information from the same teacher when they are told, "don't smoke," or "don't drink while driving,"or "don't take drugs," or, most recently, "don't put yourself at risk for AIDS." Thus the net result of the excessive warnings about eclipse eye safety may be negative, with people put at increased risk over the risk they would have had by merely being properly advised about safe ways to observe the eclipse.

Solar eclipses provide a tremendous opportunity for us astronomers and astronomical educators to reach millions of people to show them how interesting the heavens are, and what real science as opposed to pseudoscience can do. Through planning and proper diffusion of interesting and precise materials to the public, we can bring hundreds of millions of people into contact not only with astronomy but also with the capabilities of science in general. 
Acknowledgments

My recent work at solar eclipses has been supported by the U.S. National Science Foundation through its Atmospheric Sciences Division (grant ATM- 9207110), its Education Division (grant DUE-9351279), and its Astronomy Division through its Instrumentation Program (grant AST-9512216); the National Geographic Society through its Committee on Research and Exploration (grant 5190-94); and the Keck Northeast Astronomy Consortium. I also thank the Bronfman Science Center and the Safford Fund at Williams College for their support of the efforts of my students and me.

\section{REFERENCES}

Pasachoff, J.M., and Covington, M., 1993, The Cambridge Eclipse Photography Guide, Cambridge University Press;

WILliams, S., 1996, UK Solar Eclipses from Year 1, Clock Tower Press, Leighton Buzzard,UK. 